\title{
Fiber-bundle model with time-dependent healing mechanisms to simulate progressive failure of snow
}

\author{
Achille Capelli, ${ }^{1, *}$ Ingrid Reiweger, ${ }^{2}$ Peter Lehmann, ${ }^{3}$ and Jürg Schweizer ${ }^{1}$ \\ ${ }^{1}$ WSL Institute for Snow and Avalanche Research SLF, Flüelastrasse 11, 7260 Davos Dorf, Switzerland \\ ${ }^{2}$ BOKU University of Natural Resources and Life Sciences, Department of Civil Engineering and Natural Hazards, \\ Institute of Mountain Risk Engineering, Peter-Jordan-Strasse 82, 1190 Wien, Austria \\ ${ }^{3}$ Soil and Terrestrial Environmental Physics, Department of Environmental Systems Science, ETH Zurich, Zurich, Switzerland
}

(Received 6 February 2018; published 15 August 2018)

\begin{abstract}
Snow is a heterogeneous material with strain- and/or load-rate-dependent strength. In particular, a transition from ductile-to-brittle failure behavior with increasing load rate is observed. The rate-dependent behavior can partly be explained with the existence of a unique healing mechanism in snow that stems from its high homologous temperature (temperature close to melting point). As soon as broken elements in the ice matrix get in contact, they start sintering and the structure may regain strength. Moreover, the ice matrix is subjected to viscous deformation, inducing a relaxation of local load concentrations and, therefore, further counteracting the damage process. Ideal tools for studying the failure process of heterogeneous materials are the fiber-bundle models (FBMs), which allow investigating the effects of basic microstructural characteristics on the general macroscopic failure behavior. We present an FBM with two concurrent time-dependent healing mechanisms: sintering of broken fibers and relaxation of load inhomogeneities. Sintering compensates damage by creating additional intact, load-supporting fibers which lead to an increase of the bundle strength. However, the character of the failure is not changed by sintering alone. With combined sintering and load relaxation, load is distributed from old stronger fibers to new fibers that carry fewer load. So as we additionally incorporated load redistribution to the FBM, the failure occurred suddenly without decrease of the order parameter — describing the amount of damage in the bundle — and without divergence of the fiber failure rate. Moreover, the $b$ value, i.e., the power-law exponent of frequency-magnitude statistics of fibers breaking in load redistribution steps, at failure converged to $b \approx 2$, a value higher than that of a classical FBM without healing $\left(b=\frac{3}{2}\right)$. These results indicate that healing, as the combined effect of sintering and load relaxation, changes the type of the phase transition at failure. This change of the phase transition is important for quantifying or predicting the failure (e.g., by monitoring acoustic emissions) of snow or other materials for which healing plays an important role.
\end{abstract}

DOI: 10.1103/PhysRevE.98.023002

\section{INTRODUCTION}

Failure of heterogeneous materials by gradually increasing load is a progressive process which starts from damage at the microscale. Growth and interaction of the microstructural damage leads to the breakdown of the macroscopic system [1]. Fiber-bundle models (FBM) are widely used to study the failure process of heterogeneous materials [2]. In FBMs a complex behavior of the system arises from the interaction of multiple single elements (fibers) with heterogeneous properties (strength), which obey simple rules (e.g., elastic deformation). Therefore, the FBM models allow investigating the influence of local microscopic mechanisms on the (global) failure of the whole system. FBMs are often used to study material failure in the context of statistical physics and the analogy to phase transitions and critical phenomena. A variety of modifications of FBMs have been proposed to study the failure of different types of heterogeneous materials (e.g., [2,3]).

*achille.capelli@slf.ch

Published by the American Physical Society under the terms of the Creative Commons Attribution 4.0 International license. Further distribution of this work must maintain attribution to the author(s) and the published article's title, journal citation, and DOI.
Snow, a highly porous matrix of ice, is an example of a heterogeneous material with a peculiar failure behavior: if the load is applied rapidly, snow fails in a brittle manner, while if loaded slowly, it shows ductile failure behavior [4-6]. Postpeak strain softening behavior is typically observed for displacement-controlled experiments at low strain rates [4,5]. In addition, snow strength and strain at failure are higher for lower strain rates $[4,5]$. For all strain rates, snow fails at strain and stress values that are much higher than the linear elastic limit [7]. This rate dependence of the failure behavior is also reflected in the acoustic emission (AE) response of snow (e.g., $[8,9])$. The AE response in turn is used for measuring precursors of material breakdown and to predict the time of failure. A competition between damage and healing has been proposed as an explanation of the rate-dependent properties of snow $[10,11]$. Snow on the ground in its natural environment typically has a temperature close to its melting point, i.e., it has a high homologous temperature. The high homologous temperature enables the growth of new bonds between ice crystals as two crystals come in contact. The process of new bond formation between contacting snow crystals is called sintering [12]. Alternatively, it has been suggested that the viscous deformation of ice (creep) and the ductile-to-brittle transition for the failure behavior of ice are responsible for the ductile-to-brittle transition of the failure behavior of snow 
[13]. As ice creep can also induce relaxation of local load concentrations, preventing the initiation of a macroscopic failure, it can be considered as a healing mechanism countering the damage process.

We propose a combination of the two mechanisms (sintering and load relaxation) to be responsible for the rate-dependent failure behavior of snow. Moreover, we suggest that the FBM is an ideal tool to study the effect of the combination of sintering and viscous deformation inducing load relaxation during the failure of heterogeneous materials. In the classical version of the FBM, the fibers fail irreversibly when a stress or strain threshold is reached. Different versions of FBMs with healing of broken fibers have previously been proposed to reproduce the mechanical behavior of different materials. For example, in the stick-slip FBM $[14,15]$ the fibers recover strength immediately after failure. The stick-slip FBM exhibits a plastic macroscopic deformation behavior despite the brittle constituents. Similar models have been proposed for modeling the acoustic emission response produced by rearrangement of the force chain networks in granular materials $[16,17]$. Fan et al. [18] used a FBM with time-dependent healing of broken fibers to reproduce healing of soil strength and root reinforcement after rainfall events for a landslide model at hillslope scale. Bagchi and Mohanty [19] modeled species extinction; their model is equivalent to a FBM with a simple probability for broken fiber regeneration and can be solved analytically. For snow Reiweger et al. [20] proposed a FBM with time-dependent healing of broken fibers and found good agreement with results of strain-controlled shear experiments; the FBM calculations reproduced the well-known strain-ratedependent mechanical behavior of snow, including strain softening for slow strain rates. We will build the sintering part of our model on [18] but include as well the load relaxation, as it may occur during creep deformation. Creep rupture of fibrous materials has been modeled with two different versions of FBMs [21-23]: with viscoelastic fibers following the Kelvin-Voigt model (spring and dashpot parallel), and with elastic fibers, which became Maxwell elements (spring and dashpot in series) after failure. Both models showed a transition with increasing load from a stable partially failed state to an unstable state that ends with failure. Faillettaz and Or [24] studied the roles of spatial correlation and load redistribution rules in failure statistics and global failure of the FBM and showed that the FBM failure mode varies with increasing spatial correlation length and load-sharing rules.

So far, no FBM with both time-dependent healing of broken fibers as well as load relaxation has been presented, and the combined effect of these two time-dependent healing mechanisms on the type of transition at failure has not been investigated yet. We therefore developed a version of the FBM with two time-dependent healing mechanisms: (a) sintering of broken fibers and (b) relaxation of load inhomogeneities due to viscous deformation. We aim to analyze the effect of the two healing mechanisms on the fiber-bundle failure characteristics in the context of critical phenomena. We show that the combination of sintering and load relaxation significantly changes the character of the failure. Finally, we discuss the implications of our findings for the study of failure of heterogeneous materials such as snow.

\section{THEORETICAL CONSIDERATIONS OF FBM MODEL WITH HEALING}

The FBM consists of $N$ parallel fibers with heterogeneous strengths (we used a Weibull distribution). The load $\sigma$ on the fibers is increased stepwise to the strength of the weakest intact fiber that then fails (adaptive load-controlled mode). The load of the broken fiber is redistributed equally to the intact fibers (called "equal" or "democratic load" sharing). This load-sharing rule is equivalent to stiff clamping plates at the end of the fibers and leads to a range of interaction equivalent to the size of the entire bundle. The load redistribution may cause the failure of other fibers; their load is subsequently redistributed, possibly causing a cascade or avalanche of fiber failures. The avalanche stops when the load redistribution no longer causes any fiber to fail. At that point the external load is increased to the strength of the next weakest fiber. The process is repeated until all intact fibers fail within a final avalanche.

For the classical FBM (as described above) with forcecontrolled load, democratic load sharing, and without healing mechanisms, other authors have reported the following results (see reviews, e.g., $[3,25]$ ): The avalanche size $S$ (number of broken fibers) is power-law distributed $P(S) \approx S^{b}$ with $b=$ $5 / 2$ for all avalanches. If just the avalanches near failure are considered, the avalanche size is still power-law distributed but has a lower exponent with $b=3 / 2$. Moreover, the fiber failure rate $\frac{d S}{d \sigma}$ (number of breaking fibers per unit stress increase) diverges when approaching failure with $\frac{d S}{d \sigma} \approx\left(\sigma_{\mathrm{c}}-\sigma\right)^{-\alpha}$ with exponent $\alpha=1 / 2$ and critical load $\sigma_{\mathrm{c}}$ (i.e., the load when all fibers break). The order parameter $O(\sigma)=U\left(\sigma_{\mathrm{c}}\right)-U(\sigma)$ is defined as the difference between the fraction of broken fibers $U$ at any load $\sigma$ and at the critical load. $U\left(\sigma_{\mathrm{c}}\right)$ is the fraction of broken fibers immediately before the bundle fails and is smaller than 1 . The order parameter $O(\sigma)$ continuously decreases towards zero at failure with $O=\left(\sigma_{\mathrm{c}}-\sigma\right)^{\kappa}$ with exponent $\kappa=1 / 2$. In other words, towards failure the fiber bundle shows a continuous transition from a partially damaged state to a completely failed state. This behavior corresponds to a phase transition of second order.

In the present study, we assume the strength of the fibers to follow a Weibull distribution,

$$
p(\sigma \mid \mu, k)=k \mu^{-k} \sigma^{k-1} e^{-\left(\frac{\sigma}{\mu}\right)^{k}},
$$

with the shape factor $k=1.1$ (e.g., [26]). We used the Weibull distribution because it is commonly used for statistical fracture models. The failure behavior of the classical FBM is not influenced by the type of distribution and shape factor $k$ [3,27]. The mean fiber strength is set to 1 , adjusting the scale factor $\mu$ accordingly. We supplemented the classical FBM with two time-dependent healing mechanisms: resintering of broken fibers and viscous relaxation of local load concentrations.

\section{A. Sintering}

We introduce the possibility of failed fibers to regain full strength by sintering [see Fig. 1(a)]. The model works as follows (see flowchart of Fig. 2). As in the classical FBM, the load is increased incrementally and the load released from failed fibers will be redistributed to all intact neighbors. At each time step (or load step) we know the load on each fiber 

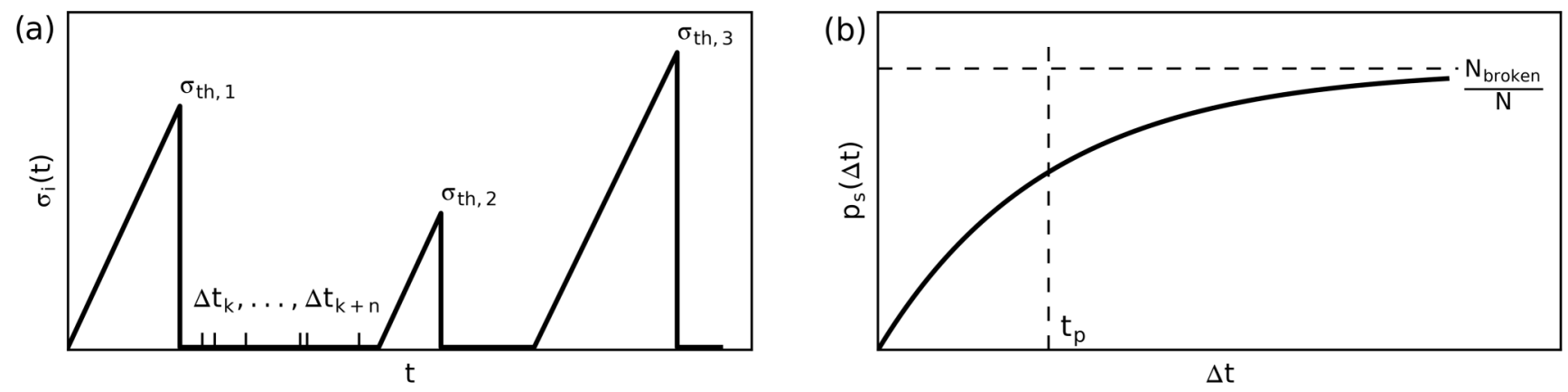

(c)

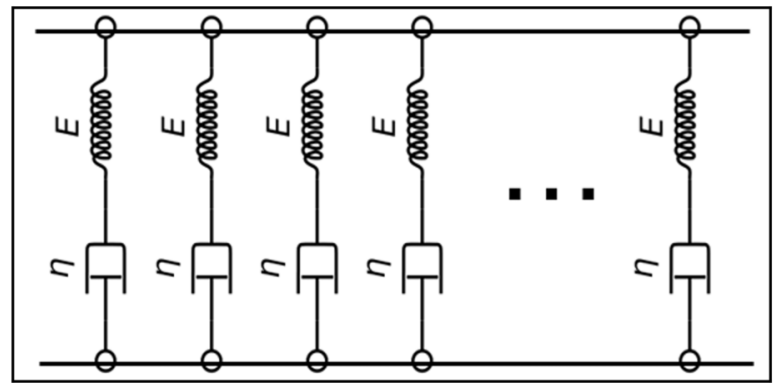

(d)

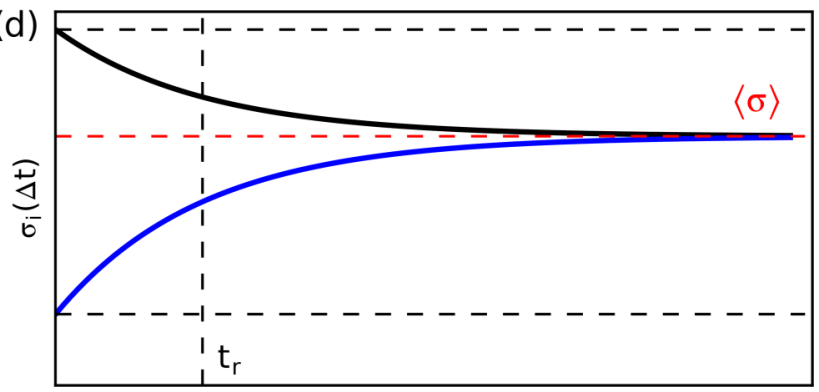

$\Delta \mathrm{t}$

FIG. 1. (a) Example of the loading of a single fiber for the FBM with sintering. Initially the load is increased until the strength $\sigma_{\text {th }, 1}$ is reached and the fiber fails. After a certain number of load steps (depending on the sintering probability $p_{s, i}$ ) the fiber sinters and gets a new strength $\sigma_{\mathrm{th}, 2}$ independent of the previous strength; subsequently, it may fail and sinter again. (b) Dependence of the sintering probability $p_{s, i}$ [Eq. (2)] on the load step length in time $\Delta t$. The speed of the sintering process is controlled by the sintering time $t_{\mathrm{p}}$. (c) Schematic representation of the FBM with load relaxation. Each fiber can be represented by a dashpot and a spring in series (Maxwell element). (d) Time evolution of the load on the single fibers in case of load relaxation [Eq. (7)] with mean load $\langle\sigma\rangle$ shown in red. The load decreases if it is higher than the mean load (black line) or increases if it is lower (blue line), in both cases converging to the mean load. The speed of the load relaxation is controlled by the characteristic loading time $t_{r}$. All times can be transformed to loads (e.g., $t_{\mathrm{p}} \rightarrow \sigma_{\mathrm{p}}$ ).

and thus we know how much load is added until the next fiber breaks. Thus for a given load rate (we look at the load rate dependency of failure patterns) we know how much time $\Delta t$ it will take until the next fiber breaks. (This time step is not constant but depends on how much load is needed to break the next fiber.) During this time period $\Delta t$ broken fibers have a chance to heal, as described next.

Each time the load is increased, the broken fibers have a certain probability to sinter. The strength of the new fibers is independent of the previous strength and is drawn from the initial strength distribution. (Such a rule is called annealed disorder, in contrast to quenched disorder, where the new fiber would obtain the same strength as before the failure.) The load on the resintered fibers is zero immediately after sintering and increases at the next load step with the external load or due to load redistribution. (So within one time step $\Delta t$ the healed fiber does not take load and is thus not affecting the time it takes until the next fiber breaks.) The adaptive loading mode causes load steps of variable size. Assuming a constant loading rate $\dot{\sigma}=\frac{d \sigma}{d t}$ results in time intervals of variable length $\Delta t$. The probability $p_{\mathrm{s}, i}$ that a given broken fiber $i$ resinters during the time interval $\Delta t$ depends on the number of broken fibers and on $\Delta t$ :

$$
p_{\mathrm{s}, i}(\Delta t)=\left(1-e^{-\frac{\Delta t}{t_{\mathrm{p}}}}\right) \frac{N_{\text {broken }}}{N},
$$

where $t_{\mathrm{p}}$ is the characteristic sintering time [Fig. 1(b)]. Therefore, accounting for all fibers, the portion of new fibers created during the time interval $\Delta t$ is

$$
p_{\mathrm{s}}(\Delta t)=\frac{1}{N} \sum_{i} p_{\mathrm{s}, i}(\Delta t)=\left(1-e^{-\frac{\Delta t}{t_{\mathrm{p}}}}\right)\left(\frac{N_{\text {broken }}}{N}\right)^{2} .
$$

The sintering probability is dependent on the number of broken fibers because we assume that two fiber ends are necessary to form a new bond. Hence, the probability of forming a new fiber increases with the amount of broken fiber ends available. This model assumption for sintering has been previously used to describe the mechanical behavior of snow $[20,28]$.

We based our sintering model on the mechanism introduced by Reiweger et al. [20] for a displacement-controlled version of a FBM. Due to the variable size of the load steps, we substitute the constant maximum sintering probability of [20] with the time-dependent sintering probability $p(\Delta t)=1-e-\left(\Delta t t_{\mathrm{p}}\right)$. Moreover, Reiweger et al. [20] introduced a second time dependence with the assumption that the resintered fibers do not reach their final strength immediately at failure, but that the strength increases with time and reaches the final strength only after the time $t_{\mathrm{s}}$. We assume that the resintered fibers gain full strength immediately after sintering. This means that a single time variable controls the sintering process and the complexity of the model is reduced. Our assumption is justifiable, since the formation of initial bonds between ice crystals in snow takes place almost immediately $[29,30]$. The further increase of bond strength in snow (resulting in an increase of strength 


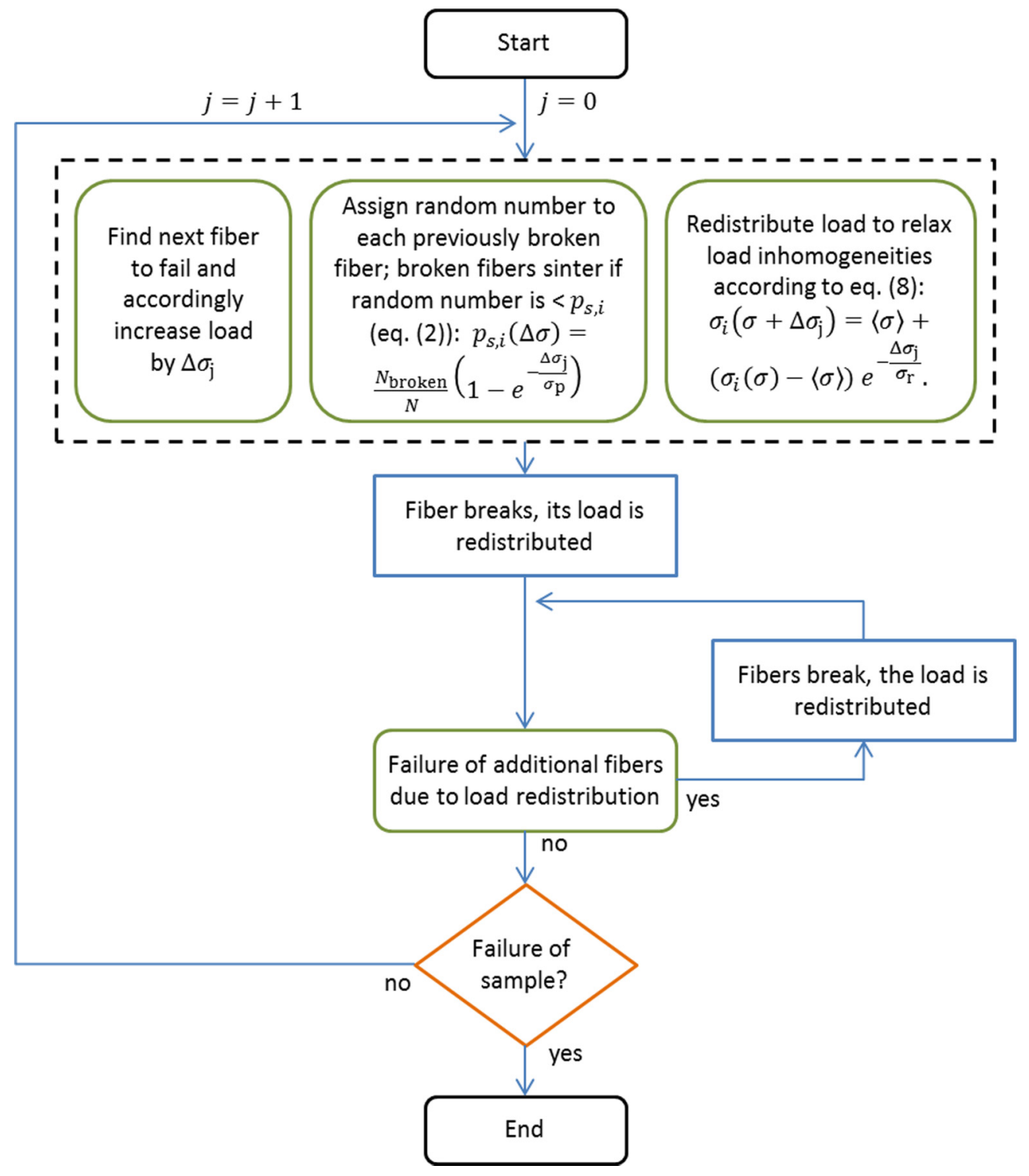

FIG. 2. Flowchart illustrating the FBM algorithm. The index $j$ indicates the load steps.

and elastic modulus) occurs at a time scale beyond the time scale of the failure process we consider in the model [31,32].

The load $\sigma$ and the time $t$ are related to $t=\frac{\sigma}{\dot{\sigma}}$. Therefore, the characteristic time $t_{\mathrm{p}}$ can be substituted with the characteristic sintering load $\sigma_{\mathrm{p}}=\dot{\sigma} t_{\mathrm{p}}$. Hence the exponential terms in Eq. (2) can be written as $e^{-\frac{\Delta t}{t_{\mathrm{p}}}}=e^{-\frac{\Delta \sigma}{\dot{\sigma} t_{\mathrm{p}}}}=e^{-\frac{\Delta \sigma}{\sigma_{\mathrm{p}}}}$, and the loading rate and the time can be eliminated, reducing the number of parameters and the complexity of the model. The characteristic sintering load $\sigma_{\mathrm{p}}$ represents the load increase over which the probability of a fiber to sinter is $1-\frac{1}{e}$ and it determines the speed of the sintering process in dependence of the load increase. Figure 3(a) shows a snapshot of the state of the FBM with sintering during a load step.

\section{B. Load relaxation}

The second mechanism that may support healing by removing load from loaded fibers is the load relaxation between the fibers during each time step $\Delta t$. The heterogeneous load redistribution stems from the sintering generating fibers that are less loaded. To model the redistribution between fibers with different loads, we assume that the fibers are viscoelastic and each fiber can be described by a Maxwell element [spring and a dashpot connected in series, Fig. 1(c)]. The corresponding constitutive equation for a fiber $i$ is given by

$$
\dot{\epsilon}_{i}=\frac{\sigma_{i}}{\eta}+\frac{\dot{\sigma}_{i}}{E},
$$

where $\sigma_{\mathrm{i}}$ is the stress on a single fiber, $E$ is the fiber's elastic modulus, and $\eta$ its viscosity. Since we assume that the fibers are connected by two rigid plates, all fibers experience the same strain $\epsilon=\frac{\sigma}{E}$. For simplicity we assume that for the short time $\Delta t$ period the change in strain is small and Eq. (4) becomes

$$
\dot{\epsilon}_{i}=0=\frac{\sigma_{i}}{\eta}+\frac{\dot{\sigma}_{i}}{E}
$$

with the solution

$$
\sigma_{i, \mathrm{rel}}(t+\Delta t)=\sigma_{\mathrm{i}}(t) e^{-\frac{E}{\eta} \Delta t}
$$




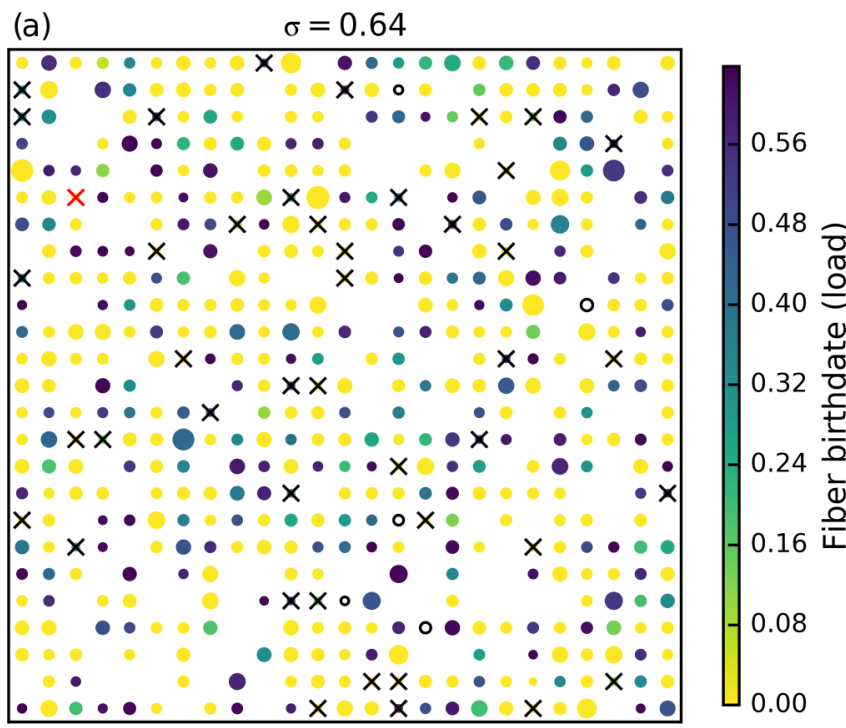

(b) $\sigma=0.64, \Delta \sigma=0.001$

FIG. 3. Snapshot of the FBM for a single load step in the form of 2D maps of the fiber bundle illustrating the sintering and load relaxation processes. For the full time evolution see Supplemental Material [42]. (a) The red cross indicates the initially failed fiber and the black crosses the subsequently failing fibers of the current avalanche. The open circles show the fibers that are sintering during the current load step. The size of the dots indicates the strength of the intact fibers and their color shows the age with yellow representing the fibers that did not fail so far and dark blue the most recently sintered fibers. (b) Representation of the load relaxation mechanism. The size of the dots shows the load carried by a single fiber; the color indicates the load change due to relaxation for the current step (yellow decrease of load and green/blue increase of load). Both figures show the same load step $(\sigma=0.64)$ with a load increase of $\Delta \sigma=0.001$ for a FBM of size $N=25 \times 25, \sigma_{\mathrm{p}}=0.015$, and $\sigma_{\mathrm{r}}=0.1$. The two-dimensional representation of the fiber bundle does not reproduce a "real" spatial distribution of the fibers, since by applying democratic load sharing the spatial position of the fibers becomes irrelevant.

Equation (6) describes the relaxation of the load for a single fiber. Since the external load on the fiber bundle is constant, the load $\sigma_{i, \text { rel }}$ on fiber $i$ decreases and the difference $\Delta \sigma_{i, \text { rel }}=$ $\sigma_{i}(t)-\sigma_{i, \text { rel }}(t+\Delta t)$ must be distributed to the other surviving fibers. Therefore, the total load on fiber $i$ after the time $\Delta t$ is

$$
\begin{aligned}
\sigma_{i}(t+\Delta t) & =\sigma_{i, \text { rel }}(t+\Delta t)+\frac{1}{N_{S}} \sum_{k}^{N_{\text {intact }}} \Delta \sigma_{k, \text { rel }} \\
& =\sigma_{i}(t) e^{-\frac{E}{\eta} \Delta t}+\frac{1}{N_{S}} \sum_{k}^{N_{\text {intact }}}\left(\sigma_{k}(t)\left[1-e^{-\frac{E}{\eta} \Delta t}\right]\right) \\
& =\sigma_{i}(t) e^{-\frac{E}{\eta} \Delta t}+\langle\sigma\rangle\left[1-e^{-\frac{E}{\eta} \Delta t}\right] \\
& =\langle\sigma\rangle+\left[\sigma_{\mathrm{i}}(t)-\langle\sigma\rangle\right] e^{-\frac{E}{\eta} \Delta t}
\end{aligned}
$$

where $\langle\sigma\rangle$ is the intact fiber mean load. According to Eq. (7), the load applied on the single fibers converges (relaxation) with time to the mean fiber load [see Fig. 1(d)]. The ratio between viscosity and elastic modulus determines the speed of relaxation and can be expressed as a relaxation time $t_{\mathrm{r}}=\frac{\eta}{E}$.
As in the case of sintering, a characteristic time for relaxation can be expressed and substituted with a load $\sigma_{\mathrm{r}}=\dot{\sigma} t_{\mathrm{r}}=\frac{\eta}{E}$. Equation (7) can now be written as

$$
\sigma_{i}(\sigma+\Delta \sigma)=\langle\sigma\rangle+\left[\sigma_{i}(\sigma)-\langle\sigma\rangle e\right]^{-\frac{\Delta \sigma}{\sigma_{\mathrm{r}}}} .
$$

The relaxation load $\sigma_{\mathrm{r}}$ represents the load increase over which $\frac{1}{e}$ of a fiber load is distributed to the other fibers and it determines the speed of the relaxation process in dependence of the load increase, i.e., the lower $\sigma_{\mathrm{r}}$ the faster the load relaxation. A snapshot of the fiber load redistributed through relaxation for a single load step is shown in Fig. 3(b) in the form of a 2D map.

\section{Deformation}

We assume that all fibers have the same elastic modulus $E$ and viscosity $\eta$. With democratic load sharing the load increase at each step is the same for all surviving fibers, and the bundle strain can be computed by summing up the mean strain increase $\Delta \epsilon_{j}$ for each load increase $\Delta \sigma_{j}$ up to load step $J$ with

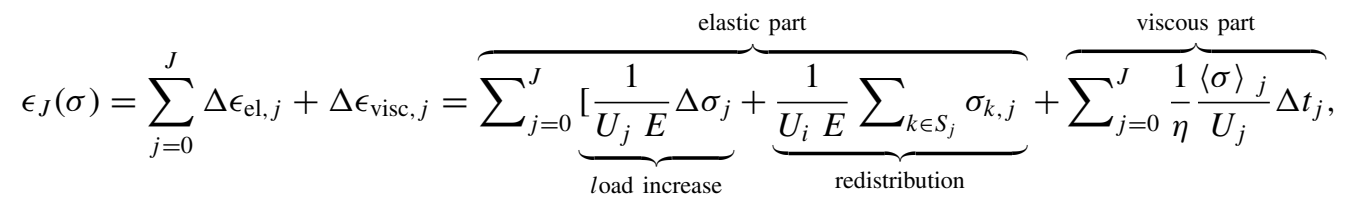



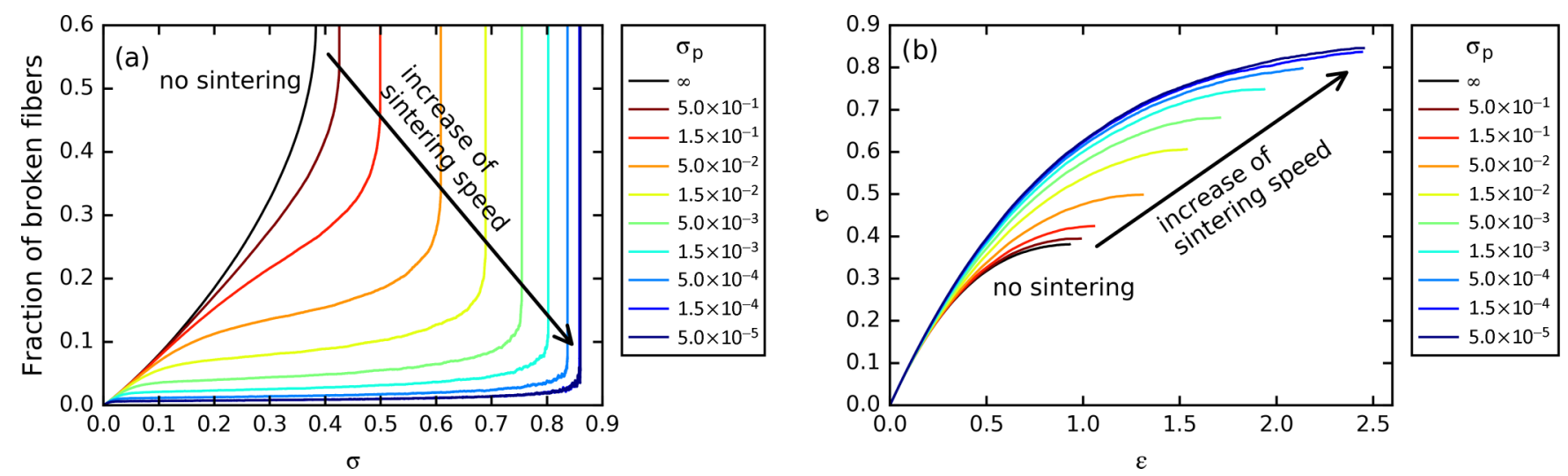

FIG. 4. (a) Fraction of broken fibers (failed minus healed) as a function of the applied load for different sintering loads. (b) Stress-strain relations of FBM for different sintering loads $\sigma_{\mathrm{p}}$. All simulations results shown here were obtained without considering relaxation $\left(i . e\right.$., $\sigma_{\mathrm{r}}=\infty$ ).

where $U_{j}$ is the fraction of intact fibers, $\sigma_{k, j}$ is the load of the fiber $k$, and $S_{j}$ is the set of fibers that failed at the load step $j$. Again, the time can be substituted with the load $\sigma$ using the relation $\sigma_{\mathrm{r}}=\dot{\sigma} t_{\mathrm{r}}=\dot{\sigma} \frac{\eta}{E}$, and we obtain

$$
\begin{aligned}
\epsilon_{J}(\sigma)= & \frac{1}{E} \overbrace{\sum_{j=0}^{J} \frac{1}{U_{j}} \Delta \sigma_{j}+\frac{\sum_{k \in S_{j}} \sigma_{k, j}}{U_{j}}}^{\text {elastic part }} \\
& +\frac{1}{E \sigma_{\mathrm{r}}} \overbrace{\sum_{j=0}^{J} \frac{\langle\sigma\rangle_{j}}{U_{j}} \Delta \sigma_{j}}^{\text {viscous part }} .
\end{aligned}
$$

The elastic part of the strain scales with $\epsilon_{\text {elastic }} \approx \frac{1}{E}$, whereas the viscous part scales with $\epsilon_{\text {visc. }} \approx \frac{1}{E \sigma_{\mathrm{r}}}$.

The unit of the elastic modulus is set to 1 and therefore the load $\sigma$ is dimensionless, with $\sigma=1$ being the mean strength of the fibers.

\section{SIMULATIONS}

To investigate the effects of sintering and load relaxation we performed simulations using nine different sintering characteristic loads $\sigma_{\mathrm{p}}$ (ranging from $5 \times 10^{-5}$ to $5 \times 10^{-1}$ ) and eight characteristic loads $\sigma_{\mathrm{r}}$ (ranging from $5 \times 10^{-5}$ to 50 ). For each set of $\sigma_{\mathrm{p}}$ and $\sigma_{\mathrm{r}}$ we computed 100 realizations of the FBM with size $N=300^{2}=9 \times 10^{4}$, and the data of the 100 realizations were merged for the computation of complementary cumulative distributions and the $b$ values. The $b$ values were computed with the PYTHON package POWERLAW [33], which employs the method described by Clauset et al. [34]. The exponents $\kappa$ and $\alpha$ were computed with a leastsquares fit of the fiber failure rate and the order parameter. To determine the exponents $\kappa$ and $\alpha$, ten realizations of size $N=10^{6}$ where used, since the quality of the fits increases with increasing the number of fibers. In the following, we first describe the effects of sintering of broken fibers without considering load relaxation and subsequently analyze the effect of load relaxation for a fixed sintering time.

\section{A. Bundle strength and stress-strain relations}

Sintering reduces the fraction of broken fibers as illustrated in Fig. 4(a). The smaller the sintering characteristic load (equivalent to short sintering times) the smaller the number of broken fibers (number of failed fibers minus the resintered fibers). If the sintering speed is high enough, after an initial increase of the fraction of broken fibers, a sort of steady state with moderate and constant increase is established. During this more or less stable state fiber failures are compensated by sintering, particularly evident for the simulations with small sintering load (smaller than about $1.5 \times 10^{-3}$ ). Shortly before failure an increase of the number of broken fibers is observed. The number of broken fibers just before global failure is lower for lower characteristic sintering loads $\sigma_{\mathrm{p}}$. Sintering of broken fibers leads to an increase of the bundle strength or critical load $\sigma_{\mathrm{c}}$, since more fibers are available to support the load [Fig. 4]. The stress-strain curves for different sintering characteristic loads $\sigma_{\mathrm{p}}$ are computed with Eq. (10) and are shown in Fig. 4(b). Sintering makes the bundle stronger and stiffer, as the strain for a given load is lower with fast sintering. For all characteristic sintering loads the strain rate diverges near failure.

The load relaxation mechanism transfers load from highly loaded fibers to less loaded fibers. For our FBM with democratic load sharing such load differences are created with the sintering of broken fibers that do not carry any load immediately after sintering. Therefore, load relaxation influences the failure behavior only in the presence of sintering. The effect of relaxation is visualized in Fig. 5(a), where the coefficient of variation of the fiber load $\mathrm{CV}=\frac{\left\langle\sigma_{\mathrm{f}}-\left\langle\sigma_{\mathrm{f}}\right\rangle\right\rangle}{\left\langle\sigma_{\mathrm{f}}\right\rangle}$ is shown as a function of the bundle load for different relaxation characteristic loads $\sigma_{\mathrm{r}}$ and a constant value of the sintering characteristic load $\sigma_{\mathrm{p}}=1.5 \times 10^{-2}$. In the absence of relaxation, the load inhomogeneity increases continuously towards failure, whereas with relaxation the load difference is reduced and even completely suppressed for very low $\sigma_{\mathrm{r}}$ values, since low $\sigma_{\mathrm{r}}$ results in faster relaxation. The effects of load relaxation on the fraction of broken fibers are shown in Fig. 6(a). Load relaxation reduces the number of broken fibers and in particular, reduces the increase of the fraction of broken fibers shortly before failure. Figure 5(b) shows the critical load $\sigma_{\mathrm{c}}$ for different relaxation characteristic loads $\sigma_{\mathrm{r}}$ and sintering characteristic loads $\sigma_{\mathrm{p}}$. The critical load $\sigma_{\mathrm{c}}$, which increases with sintering, becomes even higher with fast load relaxation [Fig. 5(b)]. More specifically, $\sigma_{\mathrm{c}}$ increases for $\sigma_{\mathrm{r}}<1$ and reaches a steady value for $\sigma_{\mathrm{r}}<10^{-2}$. The relative increase of $\sigma_{\mathrm{c}}$ reaches a maximum for $\sigma_{\mathrm{p}} \approx 10^{-2}$. 

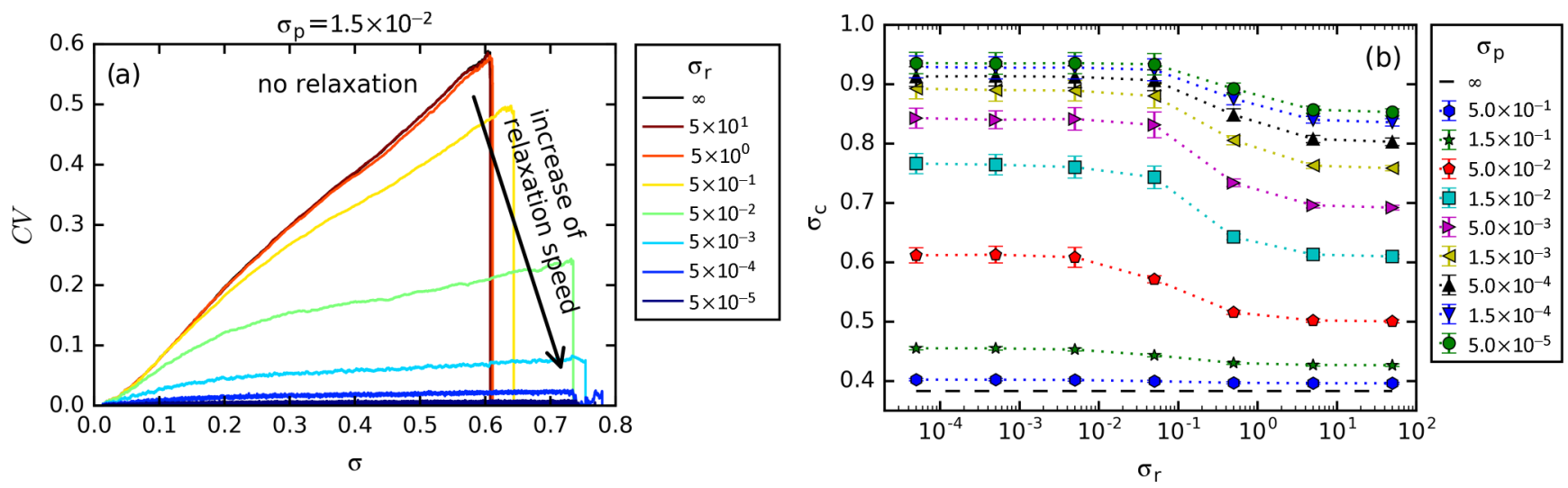

FIG. 5. (a) Coefficient of variation of the fiber load $C V=\frac{\left\langle\sigma_{i}-\left\langle\sigma_{i}\right\rangle\right\rangle}{\left\langle\sigma_{i}\right\rangle}$ as a function of the bundle load $\sigma$ for different relaxation characteristic loads $\sigma_{\mathrm{r}}$ and a constant characteristic load of sintering $\sigma_{\mathrm{p}}=1.5 \times 10^{-2}$. (b) Critical load $\sigma_{\mathrm{c}}$ as a function of the relaxation time $\sigma_{\mathrm{r}}$, and of the sintering time $\sigma_{\mathrm{p}}$.

Figure 6(b) shows the stress-strain relation for different values of $\sigma_{\mathrm{r}}$. The strain was scaled with the strain at failure $\epsilon_{\mathrm{c}}$. The scaled stress-strain curves collapse into two groups depending on which deformation type is predominant [see Eq. (10)]. The critical strain $\epsilon_{\mathrm{c}}$ is constant for low relaxation speeds, whereas it is inversely proportional to $\sigma_{\mathrm{r}}$ for high relaxation speeds [see inset Fig. 6(b) and Eq. (10)]. This means that for high relaxation characteristic loads $\sigma_{\mathrm{r}}$ the fibers deform mainly elastically, while for $\sigma_{\mathrm{r}}<2 \times 10^{-1}$ the viscous deformation is dominant. For high $\sigma_{\mathrm{r}}$ the slope of the stress-strain curve decreases monotonically to almost zero at failure. This corresponds to a divergence of the strain rate towards failure. On the other hand, for low $\sigma_{\mathrm{r}}$, the strain rate after an initial increase is almost constant up to failure.

\section{B. Avalanche size distribution}

Load redistribution of broken fibers may cause an avalanche of ruptures. The size of the rupture avalanches follows a power law characterized by its exponent the $b$ value. Figure 7 (a) shows the complementary cumulative distribution of the avalanche size $P(S)$ for different sintering characteristic loads $\sigma_{\mathrm{p}}$. Sintering causes a deviation from the original power law, with $b$ values decreasing with faster sintering [smaller characteristic load $\sigma_{\mathrm{p}}$, see inset Fig. 7(a)]. This means that for fast sintering larger avalanches are more frequent ( $b$ value is 2.1) than without sintering ( $b$ value is 2.5 ). For all scenarios the deviation from the power law for big avalanches (tail of the distribution) is due to an exponential cutoff induced by finite-size effects [25].

Figure 8 shows the $b$ value obtained for all avalanches [Fig. 8(a)] and for the avalanches close to global failure occurring in the interval $\left(\sigma_{\mathrm{c}}-\sigma\right)<10^{-3}$ [Fig. 8(b)] for different relaxation characteristic loads $\sigma_{\mathrm{r}}$ and the sintering characteristic load $\sigma_{\mathrm{p}}$. Figure 7(b) shows the evolution of the $b$ value with increasing load for representatively chosen combinations of $\sigma_{\mathrm{p}}$ and $\sigma_{\mathrm{r}}$. Without healing $\left(\sigma_{\mathrm{p}}=\infty\right)$ we obtained a $b$ value of 2.57, which is in fair agreement to the theoretical value of $b=\frac{5}{2}$ [27]. In absence of relaxation, the $b$ value decreases continuously with decreasing sintering load $\sigma_{\mathrm{p}}$ down to $b=2.15$ for $\sigma_{\mathrm{p}}=5 \times 10^{-5}$ [inset Fig. 7(a)]. A lower $b$ value indicates that the portion of large avalanches is higher.
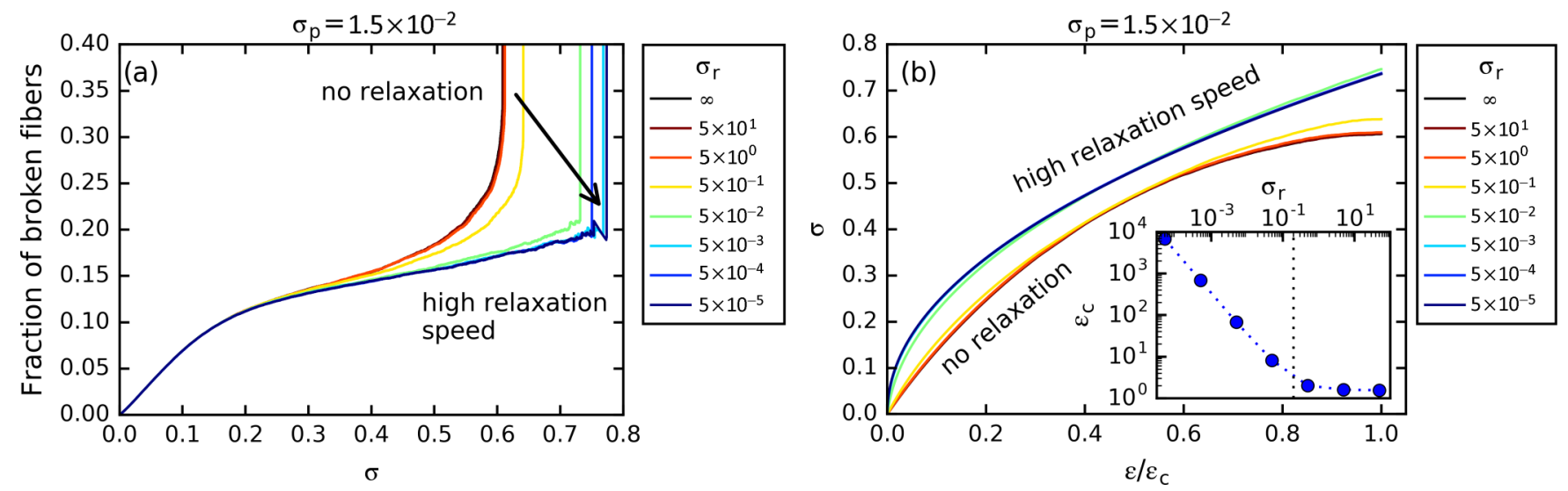

FIG. 6. (a) Fraction of broken fibers as a function of the bundle load $\sigma$ for different relaxation characteristic loads $\sigma_{\mathrm{r}}$ and a constant characteristic load of sintering $\sigma_{\mathrm{p}}=1.5 \times 10^{-2}$. (b) Stress-strain relations of FBM for different relaxation characteristic loads $\sigma_{\mathrm{r}}$ and a constant sintering characteristic load $\sigma_{\mathrm{p}}=1.5 \times 10^{-2}$. The strain was scaled with the strain at failure $\epsilon_{\mathrm{c}}$. The inset shows the strain at failure $\epsilon_{\mathrm{c}}$ as a function of the relaxation characteristic load $\sigma_{\mathrm{r}}$. 

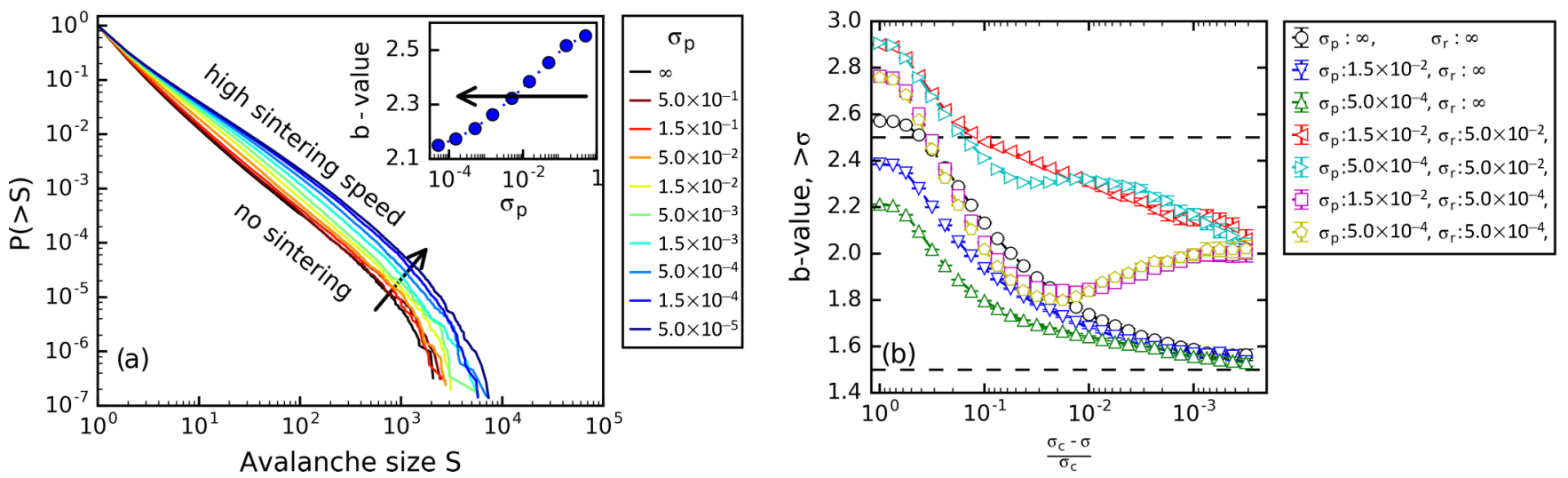

FIG. 7. (a) Complementary cumulative distribution (CCDF) of the avalanche size for different characteristic loads $\sigma_{\mathrm{p}}$ and no load relaxation. For the CCDF curves we used the data of 100 different realizations of the FBM $\left(N=9 \times 10^{4}\right)$ for each $\sigma_{\mathrm{p}}$. The inset shows the $b$ value as a function of the sintering characteristic load $\sigma_{\mathrm{p}}$. (b) Evolution of the $b$ value with increasing load $\sigma$. The $b$ value was computed from the avalanches produced at the load between $\sigma$ and $\sigma_{\mathrm{c}}$. The dashed horizontal lines mark the power exponents of the classical FBM (2.5 for entire bundle and 1.5 close to global failure).

The power-law distribution of the avalanche size of the classical FBM presents a crossover with lower $b$ value $(b=$ $1.5)$ in the vicinity of failure $[35,36]$. Without load relaxation for all sintering characteristic loads $\sigma_{\mathrm{p}}$ the $b$ value decreases with increasing load from the different initial values and converges to $b \approx 1.5$ [Figs. 8(b) and 7(b)].

Load relaxation causes an increase of the $b$ value [Fig. 8(a)]. For $\sigma_{\mathrm{r}}<2 \times 10^{-1}$ the decrease of the $b$ value caused by sintering is compensated by the load relaxation and the $b$ value is higher than in the case of the classical FBM without healing. Also, with load relaxation, a decrease of the $b$ value towards failure is observed for all combinations of $\sigma_{\mathrm{p}}$ and $\sigma_{\mathrm{r}}$. However, in the presence of load relaxation the $b$ value at failure converges to a higher $b$ value $\approx 2$ [Figs. 8(b) and 7 (b)]. Moreover, for very low values of $\sigma_{\mathrm{r}}$, the $b$ value initially decreases below 2 and later increases, converging to $b \approx 2$, whereas for the FBM without load relaxation and for moderate values of $\sigma_{\mathrm{r}}$ the decrease of the $b$ value with increasing load is monotonic. The nonmonotonic decrease of the $b$ value is observed for very high relaxation speeds independently of the sintering speed.

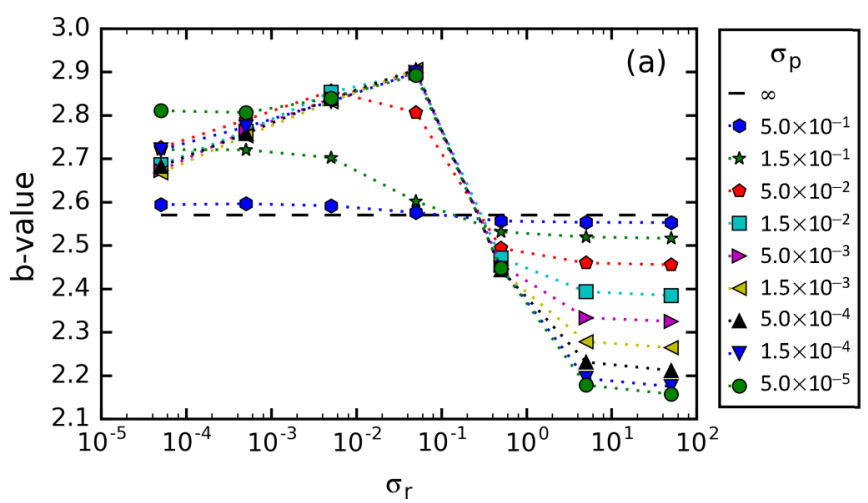

\section{Fiber failure rate}

In addition to the $b$ value of avalanche statistics, another interesting feature characteristic of the failure process and prediction is the fiber failure rate $\frac{d S}{d \sigma}$. Without relaxation the fiber failure rate increases following a power law towards global failure $\frac{d S}{d \sigma} \sim\left(\sigma_{\mathrm{c}}-\sigma\right)^{-\kappa}$ and diverges at failure [Fig. 9(a)]. The exponent $\kappa$ increases with decreasing sintering characteristic load $\sigma_{\mathrm{p}}$ from $\kappa=0.45 \pm 0.01$ (close to $\frac{1}{2}$ for classical FBM) without sintering to $\kappa=0.85 \pm 0.08$ for $\sigma_{\mathrm{p}}=$ $10^{-5}$ [Fig. 9(a), inset]. With load relaxation the divergence at bundle failure is suppressed and the fiber failure rate increases exponentially with the applied load [Fig. 9(b)].

\section{Order parameter}

For the classical FBM without healing the order parameter (difference in fraction of broken fibers at the critical load and a smaller load) converges to zero towards failure following a power law $O \sim\left(\sigma_{\mathrm{c}}-\sigma\right)^{\alpha}$ with $\alpha=1 / 2$ [3]. Figure 10(a) shows the order parameter $O$ as a function of $\sigma$ and the exponent $\alpha$ for the FBM with sintering and no relaxation. The

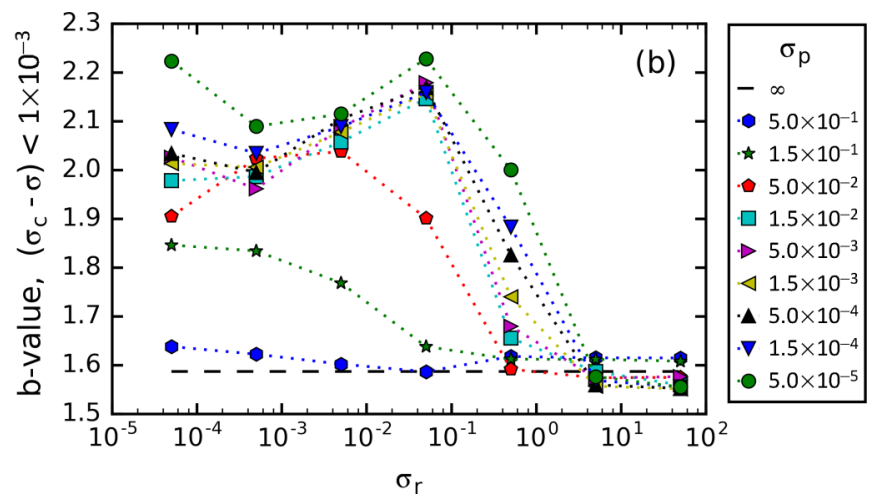

FIG. 8. Avalanche-size $b$ value for different relaxation characteristic loads $\sigma_{\mathrm{r}}$ and of the sintering characteristic load $\sigma_{\mathrm{p}}$. (a) $b$ value for all avalanches, and (b) just for the avalanches with $\left(\sigma_{\mathrm{c}}-\sigma\right)<10^{-3}$ (close to global failure). The $b$ values are obtained by combining the avalanche-size data of 100 different realizations of the FBM $\left(N=9 \times 10^{4}\right)$ for each set of $\sigma_{\mathrm{r}}$ and $\sigma_{\mathrm{p}}$. Avalanches with size $S<4$ were not considered in the computation. 

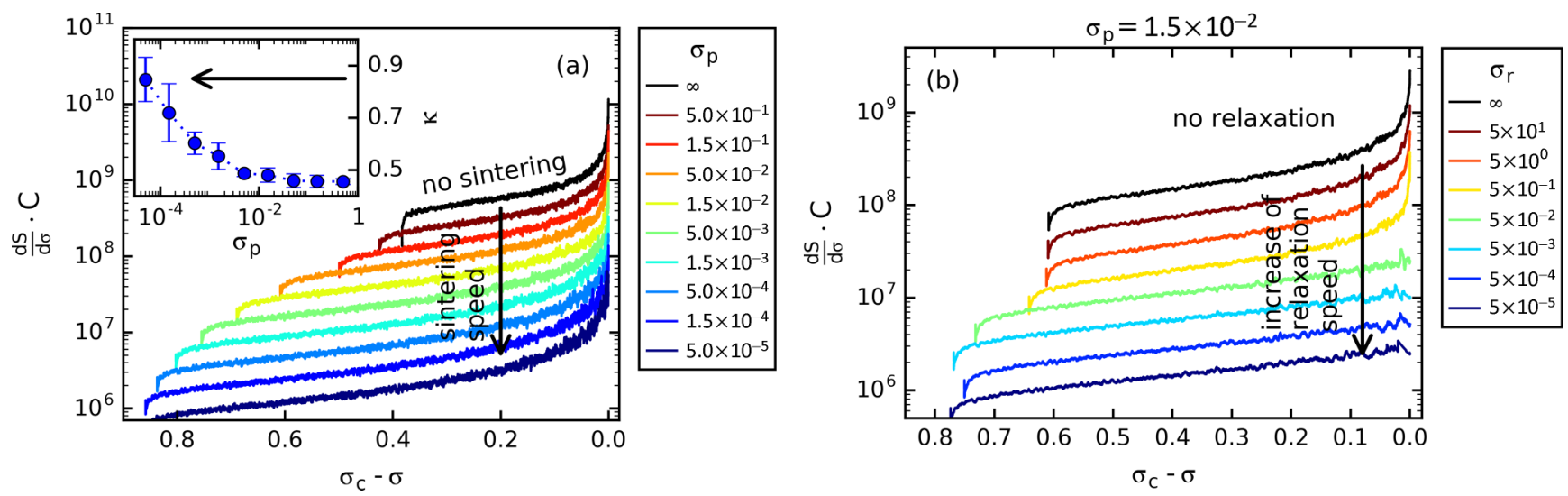

FIG. 9. (a) Evolution of the fiber failure rate towards failure for different sintering loads $\sigma_{\mathrm{p}}$ and no relaxation. The inset shows the average value of the exponent $\kappa$ with standard deviation for different $\sigma_{\mathrm{p}}$ obtained from 10 realizations of the model with size $N=10^{6}$. (b) Evolution of the fiber failure rate towards failure for different $\sigma_{\mathrm{r}}$ and constant $\sigma_{\mathrm{p}}=1.5 \times 10^{-2}$. The fiber failure rate was computed with a running window of size 1000 . In both figures, the different curves of the fiber failure rate were shifted to improve representation by multiplying $\frac{d S}{d \sigma}$ by $C=2^{n}$, with $n=(0, . ., 9)$ increasing from the lowest curve to the top one.

absolute value of $O$ is lower for lower $\sigma_{\mathrm{p}}$, since the number of failed fibers immediately before failure is lower. With sintering and no relaxation, the order parameter $O$ initially decreases rapidly with increasing load and then reaches a steady state and a more moderate decrease. Shortly before failure, $O$ decreases to zero, following a power law. The exponent $\alpha$ decreases with increasing $\sigma_{\mathrm{p}}$ from 0.5 (the expected value for classical FBM) to 0.2 [inset in Fig. 10(a)]. The power-law decreasing phase of $O$ is shorter for lower $\sigma_{\mathrm{p}}$. With load relaxation $\left(\sigma_{\mathrm{r}}<2 \times 10^{-2}\right)$ the power-law decrease of $O$ before failure is suppressed so that $O$ decreases linearly until failure [Fig. 10(b)] with $\alpha=1$.

\section{DISCUSSION}

\section{A. Sintering}

Sintering compensates the damage process and creates new fibers that support the additional load and therefore reduces the number of broken fibers at a given load. Since more fibers support the load, the strength of the bundle increases. For the same reason, the strain at the same load is lower, i.e., the bundle is stiffer [Fig. 4(b)].

In the case of sintering without relaxation the damage process of the bundle can be separated into three phases:

During the first phase the damage in the bundle (number of broken fibers) increases without any significant influence of sintering. This is due to the fact that the sintering frequency depends on the number of broken fibers [Eq. (2)] and therefore a certain damage level must be reached for the sintering process to become relevant.

In the second phase sintering and damage are in equilibrium. The number of broken fibers increases and consequently the order parameter decrease is moderate. For very low sintering characteristic loads $\sigma_{\mathrm{p}}$ the slope is almost zero.

In the third phase an acceleration of the damage process followed by catastrophic failure of the bundle is observed. This phase is characterized by a divergence of the fiber failure rate $\frac{d S}{d \sigma}$ and a power-law convergence of the order parameter $O$ to zero. The strain rate diverges as the bundle is approaching failure.
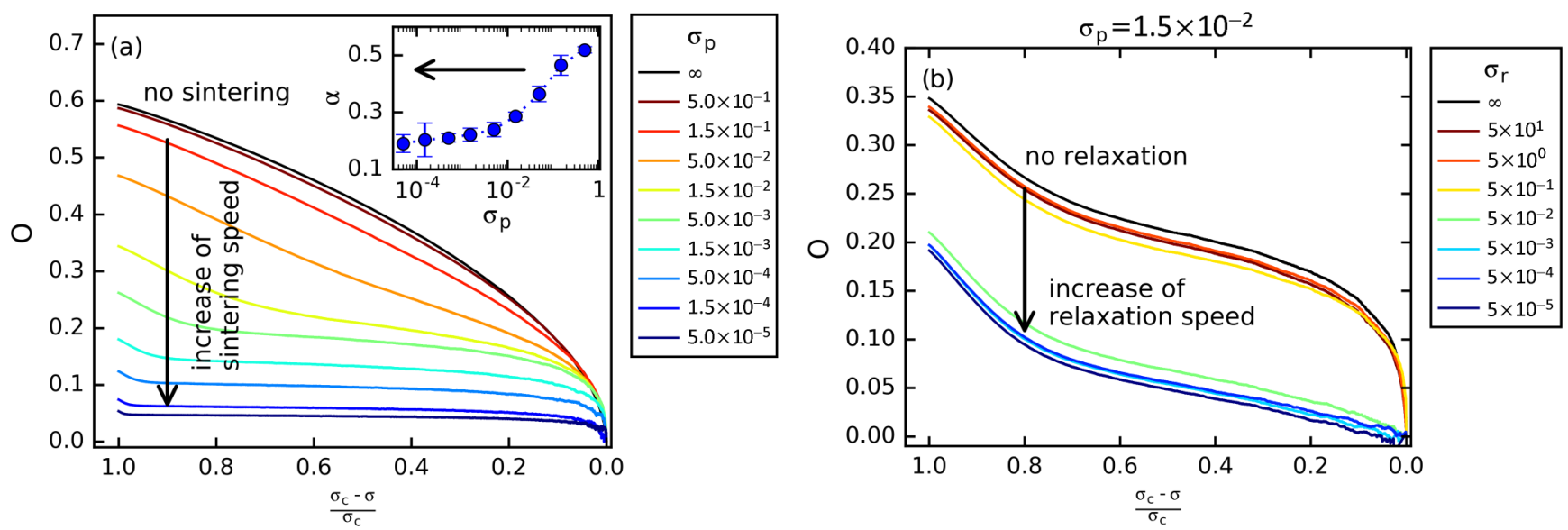

FIG. 10. (a) Evolution of the order parameter $O$ towards failure for different sintering loads $\sigma_{\mathrm{p}}$ and without load relaxation. The inset shows the average value of the exponent $\alpha$ with standard deviation for different $\sigma_{\mathrm{p}}$ obtained from ten realizations of the model with size $N=10^{6}$. (b) Evolution of the order parameter $O$ towards failure for different $\sigma_{\mathrm{r}}$ and constant $\sigma_{\mathrm{p}}=1.5 \times 10^{-2}\left(N=10^{6}\right)$. 
The lower $b$ value with lower sintering characteristic loads $\sigma_{\mathrm{p}}$ [Fig. 8(a)] indicates that the portion of large avalanches is higher with faster sintering. The higher amount of large avalanches is explained by the larger number of fibers available for failure when the load of a failed fiber is redistributed. On the other hand, the $b$ value in the vicinity of failure does not depend on $\sigma_{\mathrm{p}}$ [Fig. 8(b)], indicating that the distribution of the damage events close to failure is not influenced by sintering. Moreover, close to global failure, the sintering speed neither has influence on the order parameter $O$, which continuously converges to zero [Fig. 10(a)], nor the fiber failure rate $\frac{d S}{d \sigma}$, which diverges as the critical load is approached [Fig. 9(a)]. Those findings, in addition to the unchanged $b$ value at failure, indicate that the nature of the transition towards failure does not change with sintering. The main effect of sintering is (a) to shift the failure to a higher load, (b) to reduce the length of the damage acceleration phase, and (c) to increase the speed of both the divergence of the fiber failure rate $\frac{d S}{d \sigma}$ as well as the convergence of the order parameter $O$ (decrease of $\alpha$ and increase of $\kappa$ ).

The same $b$ value in the vicinity of failure $(b=3 / 2)$ was reported by Bagchi and Mohanty [19] for the species extinction model with a simpler healing mechanism (fixed maximum sintering probability without time dependence). They also do not report any influence of the value of the sintering probability on the type of transition. Based on our results and the results of [19], we can assume that the failure behavior is not influenced by sintering, i.e., does not depend on the sintering speed and the mechanism governing the sintering probability.

\section{B. Load relaxation}

Load relaxation reduces the load inhomogeneity by transferring load from fibers with higher load to fibers with lower load. The load inhomogeneity arises as the new fibers do not carry any load immediately after sintering. Therefore, load relaxation transfers load from the old fibers to the new ones, so stronger, older fibers survive while the new weaker fibers fail. In this way, the load is distributed more efficiently among the fibers, allowing higher loads to be sustained and thus increasing the strength of the system [in addition to the strength increase caused by the sintering, see Fig. 5(b)]. Similar results were obtained for FBM, where the load was redistributed according to the fiber strength [37]. Moreover, load transfer explains the higher $b$ value observed in the case of low relaxation loads [i.e., fast relaxation; $\sigma_{\mathrm{r}}<2 \times 10^{-1}$ see Fig. 8(a)]. Since mostly weaker fibers fail due to load transfer, the amount of load redistributed after a fiber failure is lower, reducing the probability of a large cascade of failures and, therefore, smaller avalanches prevail. The fiber's deformation develops from mainly elastic for high $\sigma_{\mathrm{r}}$ to mainly viscous for low $\sigma_{\mathrm{r}}$. Accordingly, the strain-stress curves collapse into two groups depending on the sintering speed [Fig. 6(b)]. Without load relaxation the strain rate diverges before failure, whereas for low $\sigma_{\mathrm{r}}$ the strain rate before failure is almost constant. Similarly, the fiber failure rate and the order parameter do not diverge while approaching failure $(\alpha=1)$, indicating that the load relaxation suppresses the acceleration of the damage process which is normally observed before failure or shifts the acceleration to the immediate vicinity of failure. The absence of damage acceleration before failure indicates a change in the type of transition. A further indicator of a change in the failure dynamics is the change of the $b$ value at failure from 1.5 to 2 [see Figs. 7(b) and $8(b)$ ]. For a FBM where the load of the failing fibers was redistributed to the stronger fibers, Biswas and Sen [37] reported a similar change in the type of transition with $\alpha=1$, indicating a change of the universality class. It appears that load relaxation in combination with sintering changes the failure behavior from continuous to abrupt. These model results are in accordance to results of loading experiments of snow, where at high loading rates precursors to failure were observed, whereas for slow loading rates, for which sintering and load relaxation are supposed to be most relevant, no precursors were noted [9].

\section{Consequences for failure prediction}

The power-law distribution of the avalanche size and the resulting $b$ values are scale invariant and, therefore, independent of system size. Moreover, it has been shown that the $b$ value does not depend on the type of fiber strength distribution [27]. For these reasons the change of the $b$ value towards failure is considered to be a good precursor, and the divergence of the order parameter and fiber failure rate can be used to predict the time when the bundle fails (e.g., [3]). Potentially damaging bursts or avalanches can be measured from outside without influencing the failure process (e.g., by acoustic emissions) and therefore the FBM theoretical results can be applied in practice. In the case without load relaxation, the type of transition does not change substantially with respect to the classical FBM, and accordingly, the precursors do not change much either. A minor limitation is the shorter duration of the acceleration phase, which reduces the time span before failure at which the prediction can be made. For the case with load relaxation, the failure occurs suddenly without divergence in the fiber failure rate or decrease in the order parameter; also the $b$ value converges to another value. Therefore, for a material such as snow, if loaded at a low loading rate for which sintering and load relaxation play a role, it may be difficult to find suitable precursors. Shortly before failure the fluctuations from the mean value increase for both order parameter and fiber failure rate. This increase may be used to predict failure.

\section{CONCLUSIONS AND OUTLOOK}

We presented a version of a fiber-bundle model including a combination of two healing mechanisms: (a) sintering, which is equivalent to creating new fibers with a probability depending on time and the number of broken fibers, and (b) time-dependent relaxation of load inhomogeneities between fibers. The load inhomogeneities arise since the new fibers do not carry any load. The rate of the two healing processes is regulated by the two characteristic loads $\sigma_{\mathrm{p}}$ and $\sigma_{\mathrm{r}}$. For the FBM with only sintering the bundle strength is higher than of the classical FBM, but the nature of the phase transition at failure is unchanged apart from an increase of the critical exponent $\kappa$ of the fiber failing rate and a decrease of the critical exponent $\alpha$ of the order parameter. Introducing load relaxation, the strength of the bundle further increased, since load is transferred to those fibers carrying fewer load. Moreover, for the FBM with both sintering and load relaxation, the failure occurs suddenly and the divergence of the order parameter and the fiber failure 
rate is suppressed. The $b$ value at failure changes from 1.5 to 2 for increasing relaxation speed. Hence, we show that healing as described by the combined effect of sintering and load relaxation can change the type of phase transition occurring at failure.

The failure of fibers in the FBM corresponds to the occurrence of damage in real materials, which can be monitored by acoustic emissions. The possibility of the occurrence of failure without clear precursors that we have shown must be considered for the interpretation of the acoustic emission response of snow or other materials for which healing plays an important role.

Certainly, our model represents a strong simplification of reality. Model improvements may include introducing local load-sharing rules (e.g., [2,26]) and using a more complex model for the load rheology instead of the Maxwell model (e.g., power-law creep $[13,38])$. Moreover, with a model capable of reproducing a more complex three-dimensional geometry (e.g., discrete element model DEM (e.g., $[39,40])$ the effect of the complex structure of the ice matrix could be studied. Snow failure leading to snow avalanches occurs under mixed-mode loading (shear and compression) [41]. Hence, the model could be improved by considering the more realistic load mix. On the other hand, we regard the complexity of the proposed model sufficient for investigating the basic effects of sintering and load relaxation on the failure behavior.

We consider our model as a theoretical framework appropriate for the interpretation of acoustic emissions and for the development of numerical models of heterogeneous materials for which healing is important. In particular for snow, our results are useful to explain observed differences in failure behavior during experiments with varying load or displacement rate.

\section{ACKNOWLEDGMENTS}

This project was funded by the Swiss National Science Foundation (SNF), Project No. 200021-146647. We are grateful to Dani Or for helpful comments. We also thank the anonymous reviewers for their suggestions that helped to improve this paper.
[1] A. Johansen and D. Sornette, Eur. Phys. J. B 18, 163 (2000).

[2] F. Kun, R. C. Hidalgo, F. Raischel, and H. J. Herrmann, Int. J. Fract. 140, 255 (2006).

[3] S. Pradhan, A. Hansen, and B. K. Chakrabarti, Rev. Mod. Phys. 82, 499 (2010).

[4] H. Narita, J. Glaciol. 26, 275 (1980).

[5] J. Schweizer, Ann. Glaciol. 26, 97 (1998).

[6] T. Fukuzawa and H. Narita, in Proceedings of ISSW 1992, International Snow Science Workshop, Breckenridge, CO, USA, 4-8 October 1992 (Colorado Avalanche Information Center, Denver, CO, USA, 1993), p. 171.

[7] C. Camponovo and J. Schweizer, Ann. Glaciol. 32, 44 (2001).

[8] C. Scapozza, F. Bucher, P. Amann, W. J. Ammann, and P. Bartelt, Ann. Glaciol. 38, 291 (2004).

[9] A. Capelli, I. Reiweger, and J. Schweizer, J. Glaciol. (2018), doi: 10.1017/jog.2018.43.

[10] V. de Montmollin, J. Glaciol. 28, 187 (1982).

[11] J. Schweizer, Cold Reg. Sci. Technol. 30, 43 (1999).

[12] R. O. Ramseier and C. M. Keeler, J. Glaciol. 6, 421 (1966).

[13] H. O. K. Kirchner, G. Michot, H. Narita, and T. Suzuki, Philos. Mag. A 81, 2161 (2001).

[14] Z. Halasz and F. Kun, Phys. Rev. E 80, 027102 (2009).

[15] Z. Halasz and F. Kun, Europhys. Lett. 89, 26008 (2010).

[16] G. Michlmayr, D. Or, and D. Cohen, Phys. Rev. E 86, 061307 (2012).

[17] G. Michlmayr, D. Cohen, and D. Or, J. Geophys. Res.: Solid Earth 118, 6086 (2013).

[18] L. F. Fan, P. Lehmann, and D. Or, J. Geophys. Res.: Earth Surf. 120, 1990 (2015).

[19] D. Bagchi and P. K. Mohanty, Phys. Rev. E 84, 061921 (2011).

[20] I. Reiweger, J. Schweizer, J. Dual, and H. J. Herrmann, J. Glaciol. 55, 997 (2009).

[21] F. Kun, Y. Moreno, R. C. Hidalgo, and H. J. Herrmann, Europhys. Lett. 63, 347 (2003).

[22] R. C. Hidalgo, F. Kun, and H. J. Herrmann, Phys. Rev. E 65, 032502 (2002).
[23] F. Kun, R. C. Hidalgo, H. J. Herrmann, and K. F. Pal, Phys. Rev. E 67, 061802 (2003).

[24] J. Faillettaz and D. Or, Phys. Rev. E 91, 032134 (2015).

[25] M. J. Alava, P. K. V. V. Nukalaz, and S. Zapperi, Adv Phys 55, 349 (2006).

[26] R. C. Hidalgo, Y. Moreno, F. Kun, and H. J. Herrmann, Phys. Rev. E 65, 046148 (2002).

[27] A. Hansen and P. C. Hemmer, Phys. Lett. 184, 394 (1994).

[28] F. Louchet, Cold Reg. Sci. Technol. 33, 141 (2001).

[29] D. Szabo and M. Schneebeli, Appl. Phys. Lett. 90, 151916 (2007).

[30] H. Gubler, J. Glaciol. 28, 457 (1982).

[31] A. Capelli, J. C. Kapil, I. Reiweger, D. Or, and J. Schweizer, Cold Reg. Sci. Technol. 125, 1 (2016).

[32] B. Gerling, H. Löwe, and A. van Herwijnen, Geophys. Res. Lett. 44, 11088 (2017).

[33] J. Alstott, E. Bullmore, and D. Plenz, PLoS ONE 9, e85777 (2014).

[34] A. Clauset, C. R. Shalizi, and M. E. J. Newman, SIAM Rev. 51, 661 (2009).

[35] S. Pradhan, A. Hansen, and P. C. Hemmer, Phys. Rev. E 74, 016122 (2006).

[36] S. Pradhan, A. Hansen, and P. C. Hemmer, Phys. Rev. Lett. 95, 125501 (2005).

[37] S. Biswas and P. Sen, Phys. Rev. Lett. 115, 155501 (2015).

[38] P. Barnes, D. Tabor, and J. C. F. Walker, Proc. R. Soc. London, Ser. A 324, 127 (1971).

[39] F. Kun, I. Varga, S. Lennartz-Sassinek, and I. G. Main, Phys. Rev. Lett. 112, 065501 (2014).

[40] J. Gaume, A. van Herwijnen, G. Chambon, K. W. Birkeland, and J. Schweizer, The Cryosphere 9, 1915 (2015).

[41] I. Reiweger, J. Gaume, and J. Schweizer, Geophys. Res. Lett. 42, 1427 (2015).

[42] See Supplemental Material at http://link.aps.org/supplemental/ 10.1103/PhysRevE.98.023002 for a movie of Fig. 3 during the model simulation. 